\title{
REVIEW
}

\section{The role of vascular endothelial growth factor in ossification}

\author{
Yan-Qi Yang ${ }^{1}$, Ying-Ying Tan ${ }^{1,2}$, Ricky Wong $^{1}$, Alex Wenden ${ }^{1}$, Lin-Kun Zhang ${ }^{1,3}$ and A Bakr M Rabie ${ }^{4}$ \\ Osteogenesis and angiogenesis are two closely correlated processes during bone growth, development, remodelling and repair. \\ Vascular endothelial growth factor (VEGF) is an essential mediator during the process of angiogenesis. Based on an extensive literature \\ search, which was carried out using the PubMed database and the keywords of osteogenesis, VEGF, endochondral ossification and \\ intramembranous ossification, this manuscript reviews the role of VEGF in ossification, with emphasis on its effect in endochondral and \\ intramembranous ossification. Osteogenesis and angiogenesis are closely correlated processes. VEGF acts as an essential mediator \\ during these processes. It not only functions in bone angiogenesis but also in various aspects of bone development.
} International Journal of Oral Science (2012) 4, 64-68; doi:10.1038/ijos.2012.33; published online 22 June 2012

Keywords: endochondral ossification; intramembranous ossification; osteogenesis; vascular endothelial growth factor

\section{INTRODUCTION}

Bone formation can occur through two distinct pathways, namely, endochondral ossification and intramembranous ossification. ${ }^{1-2}$ During development of some skull and facial bones or during bone repair, if bone segments are stabilized, mesenchymal precursor cells differentiate directly into bone-forming osteoblasts in a process called intramembranous ossification. This process of ossification occurs in many locations of the skeleton and includes formation of flat bones of the cranium, facial bones and addition to shafts of many other bones. During this process, osteoblasts lay down bone matrix forming spicules. Initially, these spiculae lay down trabecular bone, which is eventually replaced with lamellar. ${ }^{1}$ Alternatively, during development of long bones and vertebrae or bone repair in a biomechanically unstable environment, bone formation occurs via a cartilage intermediate in a process called endochondral ossification. ${ }^{3}$ During endochondral ossification, mesenchymal cells differentiate to chondrocytes. Vascular invasion and chondrocytes proliferate in a coordinated process that lengthens the bone. ${ }^{2}$

Osteogenesis and angiogenesis are two closely correlated processes during bone growth, development, remodelling and repair. ${ }^{4}$ Vascular endothelial growth factor (VEGF) is an essential mediator during the process of angiogenesis. Disruption of a single VEGF allele causes embryonic lethality because of defective vasculogenesis, angiogenesis and large vessel formation. ${ }^{5-6}$ Postnatal ablation of VEGF leads to abnormal organs development because of the reduced vascularisation and angiogenesis. ${ }^{7}$ Except these effects on angiogenesis, VEGF works in both processes of endochondral ossification and intramembranous ossification $^{8-12}$ and acts as an essential mediator during these processes. It is involved not only in bone angiogenesis, but also in various aspects of bone development, including chondrocyte differentiation, osteoblast differentiation and osteoclast recruitment. This article focuses predominately on the role of VEGF in the above two ossification processes.

\section{MATERIALS AND METHODS}

A literature search was carried out using PubMed and the keywordsosteogenesis; VEGF; endochondral ossification; and intramembranous ossification. Articles that only described the effect of VEGF on angiogenesis were excluded. A total of 44 references were finally included in this manuscript.

\section{VEGF AND ITS RECEPTORS}

VEGF is a specific mitogen for vascular endothelial cells. It was first identified as an endothelial-specific growth factor from bovine pituitary follicular cells by Ferrara and Davis Symth. ${ }^{13}$ The VEGF family consists of seven members, namely, placenta growth factor, VEGF-A, $-\mathrm{B},-\mathrm{C},-\mathrm{D},-\mathrm{E}$ and $-\mathrm{F}{ }^{13-14}$ They all share a common structure of eight characteristically spaced cysteine residues in a VEGF homology domain. VEGF-A is the most abundant form and is therefore commonly used in studies investigating the biological effects of VEGF. ${ }^{15}$ Thus, VEGF is commonly referred to as VEGF-A. ${ }^{16}$ Using this convention, the term VEGF mentioned in this review refers to VEGF-A.

In the osteogenesis-angiogenesis coupling, hypoxia-inducible factor is one of the key upstream regulators of VEGF. Upregulation of hypoxia-inducible factor can be induced not only by the decrease of oxygen tension, ${ }^{17-18}$ but also by other stimulus, such as insulin-like growth factor-1. ${ }^{19-20}$ Recent study indicated that changes of the level of hypoxia-inducible factor can alter the level of VEGF significantly and change the bone mass dramatically. ${ }^{21}$

The biological effects of VEGF are mediated by specific tyrosine kinase receptors (VEGFRs), i.e., Vascular endothelial growth factor receptor-1 (VEGFR-1/Flt-1) and Vascular endothelial growth factor receptor-2 (VEGFR-2/KDR). Both Flt-1 and KDR can be phosphorylated on tyrosine residues, but show different patterns of potential intracellular substrates in the in vitro immune complex kinase assay and they also mediate different cellular responses. KDR, rather than Flt-1, was found to be the major mediator of essential functions such as

${ }^{1}$ Department of Orthodontics, Faculty of Dentistry, The University of Hong Kong, Hong Kong, China; ${ }^{2}$ Unit Pakar Ortodontik, Klinik Pergigian, Poliklinik Komuniti Mak Mandin, Malaysia; ${ }^{3}$ Department of Orthodontics, Tianjin Stomatological Hospital of Nankai University, Tianjin, China and ${ }^{4}$ Private Practice

Correspondence: Dr YQ Yang, Department of Orthodontics, Faculty of Dentistry, The University of Hong Kong, 34 Hospital Road, Hong Kong SAR, China

E-mail: yangyanq@hku.hk

Received 20 February 2012; accepted 26 April 2012 
chemotaxis, mitogenesis and cytoskeletal reorganisations, whereas the functional significance of the Flt-1 remains to be determined. ${ }^{22-23}$ In addition, neuropilin-1 and neuropilin-2 are receptors for semaphorins, but they also have been shown to serve as co-receptors for VEGF. ${ }^{24}$

\section{EFFECTS OF VEGF ON BONE CELLS}

Bone is a dynamic tissue in which bone resorption and formation (bone remodelling) occur in a regulated manner under the influence of systemic hormones and local factors. Osteoclasts, the multinucleated giant cells that resorb bone, develop from haematopoietic cells of the monocyte/macrophage lineage. The osteoblast is a type of mononucleate cell, arising from osteoprogenitor cells located in the periosteum and the bone marrow that is responsible for bone formation. Besides producing osteoid, which is composed mainly of type I collagen, osteoblasts are also responsible for mineralisation of the osteoid matrix. Furthermore, osteoblasts and bone marrow stromal cells support osteoclast development via the mechanism of cell-to-cell interaction with osteoclast progenitors.

\section{EFFECTS OF VEGF ON OSTEOBLASTS}

VEGF has been implicated in various aspects of osteoblast function. Two studies have shown a dose-dependent chemoattractive effect of VEGF on primary human osteoblasts ${ }^{25}$ and human mesenchymal progenitor cells. ${ }^{26}$

In addition to its effect on cell migration, VEGF stimulates cell proliferation by up to $70 \% .{ }^{26}$ It was found that VEGF directly promotes differentiation of primary human osteoblasts in vitro by increasing nodule formation and alkaline phosphatase activity in a dosedependent manner. ${ }^{27}$ Also reported is that VEGF was expressed at low levels at the beginning of osteoblast differentiation and that its expression was strongly increased only during terminal differentiation and reached maximum expression during the period of mineralisation. ${ }^{28}$ Thus, VEGF plays an essential role in the regulation of bone remodelling by stimulating osteoblast differentiation.

Mayr-Wohlfart et al. also revealed increased expression of Flt-1 and KDR on human osteoblasts. ${ }^{25}$ An in vitro kinase assay failed to demonstrate activation of KDR upon stimulation with VEGF, which is consistent with the idea that the effect of VEGF on primary human osteoblasts is mediated via Flt- $1 .^{25}$

\section{EFFECTS OF VEGF ON OSTEOCLASTS}

Recent discovery of receptor activator nuclear factor kappa $\beta$ ligand (RANKL)-RANK interaction confirms the hypothesis that there is a direct contact between osteoblasts and osteoclasts and osteoblasts play an essential role in osteoclast differentiation. Osteoblasts express RANKL as a membrane-associated factor. Osteoclast precursors that express RANK, a receptor for RANKL, recognize RANKL through the cell-cell interaction and differentiate into osteoclasts. ${ }^{29}$ Yasuda et al. also identified another key factor for osteoclastogenesis, osteoprotegrin. ${ }^{30}$ Osteoprotegrin is produced by osteoblasts or stromal cells and binds to RANKL as a decoy receptor, therefore preventing interaction between RANKL with RANK. Thus, osteoclast differentiation is inhibited by osteoprotegrin.

Min et al. have shown that VEGF can significantly increase both the expression of RANK mRNA and surface protein in human microvascular endothelial cell lines. ${ }^{31}$ In addition, they revealed that VEGF mainly enhances RANK expression in endothelial cells through Flk-1/ KDR-protein kinase C-ERK signaling pathway, suggesting that VEGF plays an important role in modulating the angiogenic action of
RANKL under physiological or pathological conditions. Yao et al. also found that the combination of VEGF and a low dose of colonystimulating factor- 1 can upregulate the RANK expression in osteoclast precursors that is needed for osteoclastogenesis. ${ }^{32}$ Nakagawa et al. suggested that VEGF is involved in osteoclastic recruitment, differentiation and enhancement of osteoclastic bone-resorbing activity in cultured rabbit mature osteoclasts. ${ }^{33}$

\section{EFFECTS OF VEGF ON ENDOCHONDRAL OSSIFICATION}

Endochondral ossification (also referred to as intracartilaginous ossification) plays a major role in bone formation. It is an essential process during mandibular condylar growth, rudimentary formation and growth of long bones, and the healing of bone fractures. Unlike intramenbranous ossification, cartilage, an avascular tissue is present and replaced by bone during endochondral ossification. ${ }^{1}$

Endochondral ossification occurs as chondrocytes undergo proliferation, hypertrophy, cell death and osteoblastic replacement. VEGF has been indicated to serve as an important mediator during the process due to its ability to regulate blood vessel invasion (neovascularisation) into hypertrophic cartilage ${ }^{34-36}$ (Figure 1). The invading blood vessels bring undifferentiated mesenchymal cells into the mineralisation front and later differentiate into osteoblasts and engage in osteogenesis. ${ }^{34}$ Also, a recent study by Bluteau et al. for the first time showed that chondrocytes can secrete four members of the VEGF family. ${ }^{37}$ VEGF-A, -B, -C and-D were detected and upregulated at the mRNA and protein levels during chondrogenic differentiation of primary chondrocytes in the ATDC5 chondrogenic cell line. This suggests that these factors play an essential role during chondrogenesis. Zelzer et al. provided further in vivo evidence for the important

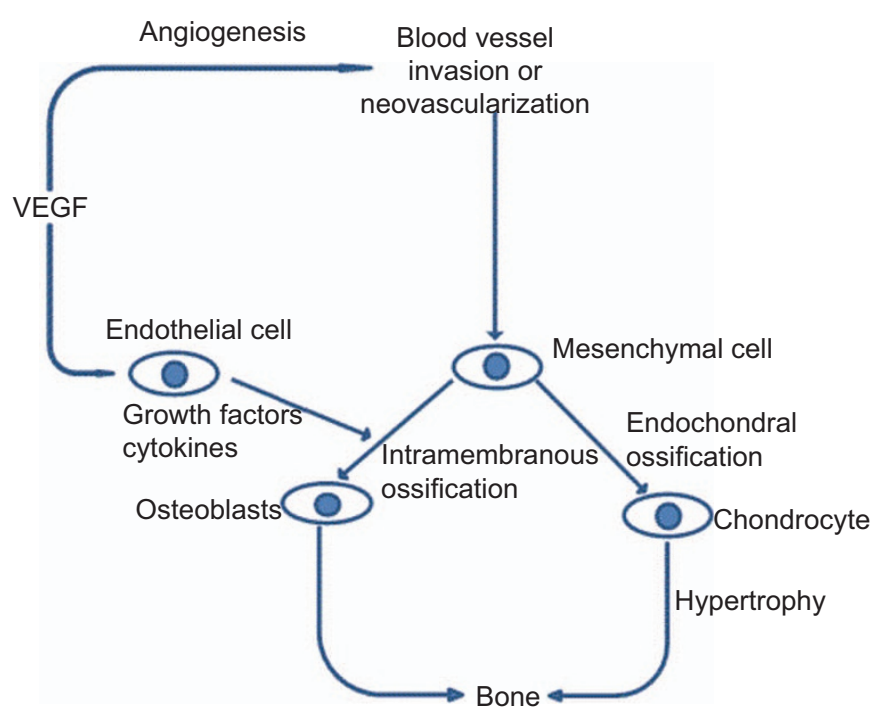

Figure 1 Schematic of effects of VEGF on angiogenesis and osteogenesis. VEGF impacts on endothelial cells and initiates the angiogenesis process which recruits original mesenchymal cells migrate to cartilage or subperioeteal connective tissue through the neonatal blood vessels. During the process of endochondral ossification, the mesenchymal cells differentiate into chondrocytes, and undergo chondrocytes hypertrophy. Then, the process of bone formation is initiated. On the other hand, during the process of intramembranous ossification, the mesenchymal cells which migrate to the subperioeteal connective tissue differentiate into osteoblasts directly and initiate the process of bone formation. VEGF upregulates the expression of growth factors and cytokines in endothelial cells and plays an important role during the process of intramembranous ossification. VEGF, vascular endothelial growth factor. 
role of VEGF in blood vessel invasion into hypertrophic cartilage during bone development. ${ }^{38}$ More importantly, they described for the first time a connection between VEGF and chondrocyte survival during skeletal development.

During mandibular growth, the condyle undergoes endochondral ossification and the condylar cartilage serves as a template for bone formation. Condylar growth involves a multistage process of cell differentiation, defined by molecules that are synthesized by cells in the condyle. ${ }^{34}$ VEGF is expressed at high levels in hypertrophic chondrocytes in the mandibular condyle of growing and adult rats. ${ }^{34,39}$ Maximum levels of VEGF expression precede the maximum level of new bone formation in the condyle. This indicates a close correlation between vascularisation and bone formation. ${ }^{35-36}$ Gene therapy explored in the condylar area by Rabie and co-workers, has shown that recombinant adeno-associated virus-mediated VEGF is an efficient delivery system to induce mandibular condylar growth ${ }^{9-11}$. Here we see a direct cause and effect. This exogenous VEGF leads to significant condylar growth. ${ }^{35-36,40-42}$

Data from Cortina-Ramírez and Chimal-Monroy suggest differential effects of VEGF on different joints during development. ${ }^{41}$ They evaluated VEGF effects on joints by implanting VEGF beads in the presumptive limb joints of chick embryos. The wrist and elbow showed partial and complete fusions. They found that VEGF inhibited joint formation when it was applied after transforming growth factor-beta.

The spheno-occipital synchondrosis is an important growth center of the craniofacial skeleton. It forms an important link between the cranial vault and facial skeleton and can influence the positions of the maxilla and mandible. ${ }^{42}$ It undergoes endochondral ossification and contributes largely to the expansion of the ossification centres and growth of the cranial base during the postnatal period. Lei et al. identified factors that regulate endochondral ossification in the spheno-occipital synchondrosis through their experiment on mice, in which they found that mechanical stress applied to the spheno-occipital synchondrosis elicits core-binding factor subunit alpha-1 expression and subsequently upregulates the expression of VEGF. ${ }^{43}$ Both factors play an important role in growth of the spheno-occipital synchondrosis.

As hypertrophic chondrocytes are avascular, these cells are a potential source of VEGF within the growth plate. To determine the role of VEGF in endochondral bone formation, Gerber et al. inactivated VEGF through the systemic administration of a soluble receptor chimeric protein (Flt-(1-3)-IgG) to 24 -day-old mice. ${ }^{44}$ Blood vessel invasion was almost completely suppressed, concomitant with impaired trabecular bone formation and expansion of the hypertrophic chondrocyte zone. Recruitment and/or differentiation of chondroclasts, which express gelatinase $\mathrm{B} /$ matrix metalloproteinase-9, and resorption of terminal chondrocytes decreased. Although proliferation, differentiation and maturation of chondrocytes were apparently normal, resorption was inhibited. Cessation of the anti-VEGF treatment was followed by capillary invasion, restoration of bone growth, resorption of the hypertrophic cartilage and normalisation of the growth plate architecture. These findings indicate that VEGFmediated capillary invasion is an essential signal that regulates growth plate morphogenesis and triggers cartilage remodelling. Thus, VEGF is a potential coordinator of chondrocyte death, chondroclast function, extracellular matrix remodelling, angiogenesis and bone formation in the growth plate. More investigates are needed to prove this hypothesis.

Healing of fractures is dependent on vascularisation of bone, which is in turn promoted by VEGF. In skeletogenesis, which is tightly linked to angiogenesis, VEGF promotes the vascularisation of the growth plate and transformation of cartilage to bone. Street et al. determined whether VEGF is required for bone repair by inhibiting VEGF activity during secondary bone healing via a cartilage intermediate/endochondral ossification in a novel mouse model. ${ }^{27}$ Femoral fractures were used as models of endochondral ossification. Fracture repair occurred in a series of events, involving an initial inflammatory phase, a soft callus phase, a hard callus phase and a remodelling phase. VEGF inhibition in mice by Flt-IgG, a soluble VEGF receptor blocker, disrupted repair of femoral fractures and impaired new bone formation. Their results provide evidence that VEGF activity is essential for conversion of soft cartilaginous callus to a hard bony callus and mineralisation in response to bone injury. Geiger et al. based on their experiment on rabbits, showed that VEGF-gene-activated matrix, led to a significant increase in vascularisation and bone regeneration in large segmental defects. ${ }^{45}$ Thus, VEGF-gene-activated matrix can serve as an appropriate tool to promote angiogenesis, osteogenesis and bone healing. Li et al. demonstrated enhanced healing of a segmental defect in the long bone of rabbits using the cell-based VEGF gene transfer without viral vectors. ${ }^{12}$ Their findings proved that this delivery method can be effective in management of clinical situations where vascularity is compromised and neovascularisation is to be encouraged, such as fractures with bone loss without the complications regarding viral vectors.

Peng et al. showed interaction between angiogenic and osteogenic factors in bone formation and bone healing. ${ }^{46}$ They discovered that the combination of VEGF and bone morphogenetic protein 4 is able to recruit more mesenchymal stem cells to enhance cell survival and to induce cartilage formation in the early stages of endochondral bone formation. Further, the beneficial effect of VEGF on bone healing was found to be dependent on the ratio of VEGF to bone morphogenetic protein 4. Table 1 summarizes the effects of VEGF on endochondral ossification.

\section{EFFECTS OF VEGF ON INTRAMEMBRANOUS OSSIFICATION}

Intramembranous ossification is a process whereby the formation of bone tissue occurs directly from connective tissue without a preliminary cartilage stage. A good example occurs in the glenoid fossa which is formed when mesenchymal cells directly differentiate into osteoblasts before ultimately forming bone. ${ }^{1}$ Experimental studies on rats have shown that mechanical strain caused by forward mandibular positioning stimulates the bone cells in the subperiosteal connective tissue of the glenoid fossa to secrete VEGF. During natural growth and forward mandibular positioning, VEGF expression and new bone formation were the highest in the posterior region of the glenoid fossa. ${ }^{35-36}$ The identification of the temporal sequence of the cellular response revealed that the mesenchymal cells in the posterior region of the glenoid fossa were oriented in the direction of the pull. ${ }^{47}$ VEGF enhances neovascularisation, which in turn increases the number of mesenchymal cells in the perivascular connective tissue (Figure 1). VEGF also stimulates the vascular endothelial cells to secrete growth factors and cytokines that influence the differentiation of mesenchymal cells to enter the osteogenic pathway and engage in osteogenesis ${ }^{48}$ (Figure 1). Furthermore, the greatest amount of VEGF expression precedes the greatest increase in new bone formation. Therefore, it has been concluded that an increase of VEGF is spatially and temporally related to the amount of new bone formation in the posterior glenoid fossa. ${ }^{35-36}$

Bone repair is a multistep process which involves migration, proliferation, differentiation and activation of several cells types. Study from Tatsuyama et al. ${ }^{49}$ on fracture repair in bone indicates that this 
Table 1 Effects of VEGF on endochondral ossification

\begin{tabular}{|c|c|c|c|}
\hline Methods & Materials & Effects & References \\
\hline \multirow[t]{12}{*}{ In vivo } & Mouse femur & Stimulated capillary invasion and bone growth & 44 \\
\hline & Rat mandibular condyle & Blood vessel invasion ( neovascularisation) into hypertrophic cartilage was regulated & 34 \\
\hline & Rat mandibular condyle & & 35 \\
\hline & & $\begin{array}{l}\text { - } \text { Promoted neovascularisation } \\
\text { - Close correlation between vascularisation and bone formation }\end{array}$ & \\
\hline & Mouse calvarial defects & $\begin{array}{l}\text { Synergistic effect between VEGF and BMP4 occurred and recruited more mesenchymal stem } \\
\text { cells to enhance cell survival and to induce cartilage formation }\end{array}$ & 46 \\
\hline & Mouse femur fractures healing model & Conversion of soft cartilaginous callus to a hard bony callus and mineralisation was induced & 27 \\
\hline & Mouse embryos & VEGF increased blood vessel invasion into hypertrophic cartilage during bone development & 38 \\
\hline & Rabbit bone defects & VEGF-GAM (gene-activated matrix) increased vascularisation and bone regeneration & 45 \\
\hline & Rat mandibular condyle & Mandibular condyle increased in size & 11 \\
\hline & Limb joints of chick embryos & $\begin{array}{l}\text { Differential effects on different joints: partial fusion on wrist joint; complete fusion on elbow } \\
\text { joint; inhibited joint formation when applied after TGF- } \beta\end{array}$ & 41 \\
\hline & Cranial base synchondroses of mice & Cbfa1 and VEGF promoted growth of the spheno-occipital synchondrosis & 43 \\
\hline & Rabbit tibial fracture defects & Healing of segmental defect in the long bone of rabbits were enhanced & 12 \\
\hline In vitro & ATDC5 chondrogenic cell line & Stimulated chondrogenic differentiation of primary chondrocytes & 37 \\
\hline
\end{tabular}

BMP4, bone morphogenetic protein 4; Cbfa1, core-binding factor subunit alpha-1; GAM, gene-activated matrix; TGF- $\beta$, transforming growth factor beta; VEGF, vascular endothelial growth factor.

process is complex, occurring through several steps and involving various growth factors including VEGF. Bone growth and bone repair are somehow regulated in a similar manner and thus, VEGF should have a similar effect during bone repair. ${ }^{8}$ Repair of large bony defects in the craniofacial region represent a major challenge for the surgeon, since autogenous graft material from patients may not be available in sufficient amounts. It has been shown that addition of VEGF to demineralized intramembranous bone matrix (DBMIM) improves the quality and quantity of newly formed bone in the grafted site, thus indicating that VEGF+DBMIM is a good graft material. It is both easily obtainable and could eliminate the need to harvest bone from the patient. Its future application, however, will require further clinical evidence.

Results from Street et al. on mice also show the role of VEGF in intramembranous ossification. ${ }^{27}$ They have shown direct bone repair through intramembranous ossification in a novel mouse model (tibial cortical bone defects). Inhibition of VEGF by Flt-IgG treatment, just as in the femoral fracture model, had distinct effects on bone healing of cortical bone defects. The persistence of unresorbed, unmineralized fracture haematoma at 7 days and the persistence of woven bone at 14 days indicate remodelling defects in Flt-IgG-treated mice. They found that the effects of Flt-IgG on intramembranous bone formation (cortical defects were more prominent at the earlier (7 days) than later (14 days) time point) were due to the fact that intramembranous ossification does not involve a cartilage intermediate. Thus, they suggested that a slow release formulation of VEGF, applied locally at the site of bone damage, can be an effective therapy to promote human bone repair.

Another example of the role of VEGF in intramembranous ossification is observed in distraction osteogenesis, a surgical therapy used in the treatment of skeletal injuries and deformities and in the correction of limb length abnormalities. ${ }^{50}$ Bone formation during distraction osteogenesis occurs primarily through an intramembranous process. Investigators observed close correlation between optimal angiogenic response and the rate of distraction and speculated that it is this characteristic that drives bone formation through the intramembranous pathway. ${ }^{51}$

To elucidate the functional role of VEGF signaling during bone formation in distraction osteogensis, Jacobsen et al. used two blocking antibodies MF-1 (anti-Flt-1) and DC101 (anti-KDR) to specifically disrupt the activities of KDR and Flt- $1 .{ }^{52}$ The results showed that both KDR and Flt-1 play important roles in neovascularisation and bone formation during distraction osteogenesis. Table 2 summarizes the effects of VEGF on intramembranous ossification.

Table 2 Effects of VEGF on intramembranous ossification

\begin{tabular}{|c|c|c|c|}
\hline Methods & Materials & Effects & References \\
\hline \multirow[t]{6}{*}{ In vivo } & Rat tibial fracture defects & VEGF enhanced fracture bone repair & 49 \\
\hline & Tibial cortical bone defects & Improved direct bone repair & 27 \\
\hline & Rat glenoid fossa & $\begin{array}{l}\text { Maximum level of VEGF expression and new bone formation in the posterior region of the glenoid } \\
\text { fossa during natural growth and forward mandibular positioning. Greatest amount of VEGF } \\
\text { expression precedes the greatest increase in new bone formation }\end{array}$ & 36 \\
\hline & Rat glenoid fossa & Secretion of growth factors and cytokines by vascular endothelial cells was enhanced & 48 \\
\hline & Rabbit parietal bone defects & Combination of VEGF and $\mathrm{DBM}_{\mathrm{IM}}$ is a good graft material & 8 \\
\hline & $\begin{array}{l}\text { Mouse tibia distraction } \\
\text { osteogenesis }\end{array}$ & $\begin{array}{l}\text { Both KDR and Flt-1 play important roles in neovascularisation and bone formation during } \\
\text { distraction osteogenesis }\end{array}$ & 52 \\
\hline In vitro & Distraction osteogenesis & Optimal angiogenic response and rate of distraction are closely related & 51 \\
\hline
\end{tabular}

DBMIM, demineralized intramembranous bone matrix; FIt-1, vascular endothelial growth factor receptor-1; KDR, vascular endothelial growth factor receptor-2; VEGF, vascular endothelial growth factor. 


\section{CONCLUSION}

The evidence from a large amount of research has shown conclusively that VEGF is able to promote ossification by either inducing neovascularisation or by directly affecting bone cells. VEGF can stimulate ossification through the two pathways of endochondral ossification and intramembranous ossification.

1 Marks SC, Hermey DC. The structure and development of bone. In: Bilezekian JP, Raisz LG, Rodan GA, editors. Principles of bone biology. San Diego: Academic Press; 1996: 3-24.

2 Kanczler JM, Oreffo RO. Osteogenesis and angiogenesis: the potential for engineering bone. Eur Cell Mater 2008; 2(15): 100-114.

3 Mandracchia VJ, Nelson SC, Barp EA. Current concepts of bone healing. Clin Podiatr Med Surg 2001; 18(1): 55-77.

4 Rabie AB. Vascular endothelial growth pattern during demineralized bone matrix induced osteogenesis. Connect Tissue Res 1997; 36(4): 337-345.

5 Carmeliet P, Ferreira V, Breier G et al. Abnormal blood vessel development and lethality in embryos lacking a single VEGF allele. Nature 1996; 380(6573): 435439.

6 Ferrara N, Carver-Moore K, Chen $\mathrm{H}$ et al. Heterozygous embryonic lethality induced by targeted inactivation of the VEGF gene. Nature 1996; 380(6573): 439-443.

7 Gerber HP, Hillan KJ, Ryan AM et al. VEGF is required for growth and survival in neonatal mice. Development 1999; 126(6): 1149-1159.

8 Emad B, Sherif EM, Basma GM et al. Vascular endothelial growth factor augments the healing of demineralized bone matrix grafts. Int J Surg 2006; 4(3): 160-166.

9 Dai J, Rabie AB. Direct AAV-mediated gene delivery to the temporomandibular joint. Front Biosci 2007; 12: 2212-2220.

10 Dai J, Rabie AB. Recombinant adeno-associated virus vector hybrids efficiently target different skeletal cells. Front Biosci 2007; 12: 4280-4287.

11 Rabie ABM, Dai J, Xu R. Recombinant AAV-mediated VEGF gene therapy induces mandibular condylar growth. Gene Ther 2007; 14(12): 972-980.

12 Li R, Stewart DJ, von Schroeder HP et al. Effect of cell-based VEGF gene therapy on healing of a segmental bone defect. J Orthop Res 2009; 27(1):8-14.

13 Ferrara N, Davis Symth T. The biology of vascular endothelial growth factor. Endocr Rev 1997; 18(1): 4-25.

14 Roy H, Bhardwaj S, Ylä-Herttuala S. Biology of vascular endothelial growth factors. FEBS Lett 2006; 580(12): 2879-2887.

15 Thomas KA. Vascular endothelial growth factor, a potent and selective angiogenic agent. J Biol Chem 1996; 271(2): 603-606.

16 Ferrara N, Gerber, HP, LeCouter J. The biology of VEGF and its receptors. Nat Med 2003; 9(6): 669-676.

17 Pouyssegur J, Dayan F, Mazure NM. Hypoxia signaling in cancer and approaches to enforce tumour regression. Nature 2006; 441(7092): 437-443.

18 Chan D, Suthphin P, Denko N et al. Role of prolyl hydroxylation in oncogenically stabilized hypoxia-inducible factor-1alpha. J Biol Chem 2002; 277(42): 4011240117.

19 Fukuda R, Hirota K, Fan F et al. Insulin-like growth factor 1 induces hypoxia-inducible factor 1-mediated vascular endothelial growth factor expression, which is dependent on MAP kinase and phosphatidylinositol 3-kinase signaling in colon cancer cells. J Biol Chem 2002; 277(41): 38205-38211.

20 Laughner E, Taghavi P, Chiles $\mathrm{K}$ et al. HER2 (neu) signaling increases the rate of hypoxiainducible factor 1alpha (HIF-1alpha) synthesis: novel mechanism for HIF-1mediated vascular endothelial growth factor expression. Mol Cell Biol 2001; 21(12): 3995-4004.

21 Wang $\mathrm{Y}$, Wan $\mathrm{C}$, Deng $\mathrm{L}$ et al. The hypoxia-inducible factor alpha pathway couples angiogenesis to osteogenesis during skeletal development. J Clin Invest 2007; 117(6): 1616-1626.

22 Ferrara N. Vascular endothelial growth factor: basic science and clinical progress. Endocr Rev 2004; 25(4): 581-611.

23 Waltenberger J, Claesson-Welsh L, Siegbahn A et al. Different signal transduction properties of KDR and Flt1, two receptors for vascular endothelial growth factor. J Biol Chem 1994; 269(43): 26988-26995.

24 Soker S, Takashima S, Miao HQ et al. Neuropilin-1 is expressed by endothelial and tumor cells as an isoform-specific receptor for vascular endothelial growth factor. Cell 1998; 92(6): 735-745.

25 Mayr-Wohlfart U, Waltenberger J, Hausser $\mathrm{H}$ et al. Vascular endothelial growth factor stimulates chemotactic migration of primary human osteoblasts. Bone 2002; 30(3): 472-477.

26 Fiedler J, Leucht F, Waltenberger J et al. VEGF-A and PIGF-1 stimulate chemotactic migration of human mesenchymal progenitor cells. Biochem Biophys Res Commun 2005; 334(2): 561-568.
27 Street J, Bao M, deGuzman L et al. Vascular endothelial growth factor stimulates bone repair by promoting angiogenesis and bone turnover. Proc Natl Acad Sci U S A 2002; 99(15): 9656-9661.

28 Deckers MM, Karperien M, van der Bent $\mathrm{C}$ et al. Expression of vascular endothelial growth factors and their receptors during osteoblast differentiation. Endocrinology 2000; 141(5): 1667-1674.

29 Katagiri T, Takahashi N. Regulatory mechanisms of osteoblast and osteoclast differentiation. Oral Dis 2002; 8(3): 147-159.

30 Yasuda $\mathrm{H}$, Shima $\mathrm{N}$, Nakagawa $\mathrm{N}$ et al. A novel molecular mechanism modulating osteoclast differentiation and function. Bone 1999; 25(1): 109-113.

31 Min JK, Kim YM, Kim YM et al. Vascular endothelial growth factor up-regulates expression of receptor activator of NF-kappa B (RANK) in endothelial cells. Concomitant increase of angiogenic responses to RANK ligand. J Biol Chem 2003; 278(41): 39548-39557.

32 Yao S, Liu D, Pan F et al. Effect of vascular endothelial growth factor on RANK gene expression in osteoclast precursors and on osteoclastogenesis. Arch Oral Biol 2006; 51(7): 596-602.

33 Nakagawa M, Kaneda T, Arakawa T et al. Vascular endothelial growth factor (VEGF) directly enhances osteoclastic bone resorption and survival of mature osteoclasts. FEBS Lett 2000; 473(2): 161-164.

34 Rabie AB, Hägg U. Factors regulating mandibular condylar growth. Am J Orthod Dentofacial Orthop 2002; 122(4): 401-409.

35 Rabie AB, Leung FY, Chayanupatkul A et al. The correlation between neovascularization and bone formation in the condyle during forward mandibular positioning. Angle Orthod 2002; 72(5): 431-443.

36 Rabie AB, Shum L, Chayanupatkul A. VEGF and bone formation in the glenoid fossa during forward mandibular positioning. Am J Orthod Dentofacial Orthop 2002; 122(2): 202-209.

37 Bluteau G, Julien M, Magne D et al. VEGF and VEGF receptors are differentially expressed in chondrocytes. Bone 2007; 40(3): 568-576.

38 Zelzer E, Mamluk R, Ferrara N et al. VEGFA is necessary for chondrocyte survival during bone development. Development 2004; 131(9): 2161-2171.

39 Xiong $\mathrm{H}$, Rabie AB, Hagg U. Neovascularization and mandibular condylar bone remodelling in adult rats under mechanical strain. Front Biosci 2005; 10: 74-82.

40 Dai J, Rabie ABM. VEGF: an essential mediator of both angiogenesis and endochondral ossification. J Dent Res 2007; 86(10): 937-950.

41 Cortina-Ramírez GE, Chimal-Monroy J. Differential effects of vascular endothelial growth factor on joint formation during limb development. Ann N Y Acad Sci 2007; 1116: 134-140.

42 Bjork A. Cranial base development. Am J Orthod 1955; 41(3): 198-225.

43 Lei WY, Wong RW, Rabie AB. Factors regulating endochondral ossification in the spheno-occipital synchondrosis. Angle Orthod 2008; 78(2): 215-220.

44 Gerber HP, Vu TH, Ryan AM et al. VEGF couples hypertrophic cartilage remodeling, ossification and angiogenesis during endochondral bone formation. Nat Med 1999; 5(6): 623-628.

45 Geiger F, Bertram H, Berger I et al. Vascular endothelial growth factor gene-activated matrix (VEGF165-GAM) enhances osteogenesis and angiogenesis in large segmental bone defects. J Bone Miner Res 2005; 20(11): 2028-2035.

46 Peng $\mathrm{H}$, Wright $\mathrm{V}$, Usas A et al. Synergistic enhancement of bone formation and healing by stem cell-expressed VEGF and bone morphogenetic protein-4. J Clin Invest 2002; 110(6): 751-759.

47 Rabie AB, Zhao Z, Shen G et al. Osteogenesis in the glenoid fossa in response to mandibular advancement. Am J Orthod Dentofacial Orthop 2001; 119(4): 390-400.

48 Shum L, Rabie AB, Hägg U. Vascular endothelial growth factor expression and bone formation in posterior glenoid fossa during stepwise mandibular advancement. $\mathrm{Am} \mathrm{J}$ Orthod Dentofacial Orthop 2004; 125(2): 185-190.

49 Tatsuyama $\mathrm{K}$, Maezawa $\mathrm{Y}, \mathrm{Baba} \mathrm{H}$ et al. Expression of various growth factors for cell proliferation and cytodifferentiation during fracture repair of bone. Eur $\mathrm{J}$ Histochem 2000; 44(3): 269-278.

50 Codivilla A. On the means of lengthening, in the lower limbs, the muscles and tissues which are shortened through deformity. Am J Orthop Surg 1905; 2: 353-369.

51 Lewinson D, Maor G, Rozen $\mathrm{N}$ et al. Expression of vascular antigens by bone cells during bone regeneration in a membranous bone distraction system. Histochem Cell Biol 2001; 116(5): 381-388.

52 Jacobsen $\mathrm{KA}, \mathrm{Al}-\mathrm{Aql} \mathrm{ZS}$, Wan $\mathrm{C}$ et al. Bone formation during distraction osteogenesis is dependent on both VEGFR1 and VEGFR2 signaling. J Bone Miner Res 2008; 23(5): 596-609.

This work is licensed under a Creative Commons Attribution-NonCommercial-NoDerivative Works 3.0 Unported License. To view a copy of this license, visit http:// creativecommons.org/licenses/by-nc-nd/3.0 